\title{
Generalized mixed equilibrium problems and quasi- $\phi$-asymptotically nonexpansive multivalued mappings in Banach spaces
}

\author{
Bashir Ali ${ }^{1 * \dagger}$ (D), Lawal Umar $2,4+$ and M. H. Harbau ${ }^{3+}$
}

\author{
*Correspondence: \\ bashiralik@yahoo.com \\ ${ }^{\dagger}$ All authors contributed equally to \\ this work. \\ 'Department of Mathematical \\ Sciences, Bayero University, Kano, \\ Nigeria \\ Full list of author information is \\ available at the end of the article
}

\begin{abstract}
In this paper, we introduce two iterative algorithms for finding a common element of the set of fixed points of a quasi- $\phi$-asymptotically nonexpansive multivalued mapping and the sets of solutions of generalized mixed equilibrium problem in Banach space. Then, we prove strong and weak convergence of the sequences to element in the mentioned set. Our results generalize and improve recent results announced by many authors.
\end{abstract}

Keywords: Fixed point, Generalized mixed equilibrium problem, Quasi- $\Phi$ -asymptotically nonexpansive multivalued mappings, Banach space

\section{Introduction}

Let $E$ be a real Banach space with norm $\|$. $\|, E^{*}$ be the dual space of $E$, and $C$ be a nonempty closed convex subset of $E$. Let $f$ be a bifunction from $C \times C$ to $\mathbb{R}$, where $\mathbb{R}$ is the set of real numbers. The equilibrium problem is to find $\hat{x} \in C$ such that

$$
f(\hat{x}, y) \geq 0, \forall y \in C .
$$

This problem was first studied by Blum and Oettli [1]. The set of solutions of equilibrium problem (1.1) is denoted by $E P(f)$ that is $E P(f)=\{\hat{x} \in C: f(\hat{x}, y) \geq 0, \forall y \in C\}$. Let $A: C \longrightarrow E^{*}$ be a nonlinear mapping. The variational inequality problem with respect to $A$ and $C$ is to find $u \in C$ such that $\langle A u, v-u\rangle \geq 0$ for all $v \in C$. The set of solutions of variational inequality problem with respect to $C$ and $A$ is denoted by $\operatorname{VI}(C, A)$. Setting $f(x, y)=\langle A x, y-x\rangle$ for all $x, y \in C$, then $\hat{x} \in E P(f)$ if and only if $\langle A \hat{x}, y-\hat{x}\rangle \geq 0$, for all $y \in C$, i.e., $\hat{x}$ is a solution of the variational inequality with respect to $A$ and $C$. Let $\varphi: C \longrightarrow \mathbb{R} \cup\{\infty\}$ be proper, convex, and lower semi-continuous, then the minimization problem of $\varphi$ is to find $x \in C$ such that $\varphi(x) \leq \varphi(y) \forall y \in C$.

The generalized equilibrium problem is to find $\hat{x} \in C$ such that

$$
f(\hat{x}, y)+\langle A \hat{x}, y-\hat{x}\rangle \geq 0, \forall y \in C .
$$

The set of solutions of (2) is denoted by

$$
G E P(f, A)=\{\hat{x} \in C: f(\hat{x}, y)+\langle A \hat{x}, y-\hat{x}\rangle \geq 0, \forall y \in C\}
$$

(c) The Author(s). 2019 Open Access This article is distributed under the terms of the Creative Commons Attribution 4.0 International License (http://creativecommons.org/licenses/by/4.0/), which permits unrestricted use, distribution, and reproduction in any medium, provided you give appropriate credit to the original author(s) and the source, provide a link to the Creative Commons license, and indicate if changes were made. 
In this paper, we are interested in solving equilibrium problem with respect to $f$ given by

$$
f(x, y)=\sum_{i=1}^{k} f_{i}(x, y), \forall x, y \in C,
$$

where $f_{i}: C \times C \longrightarrow \mathbb{R}$ are bifunctions for $i=1,2,3, \ldots k$, satisfying the following conditions $\left(A_{1}\right)-\left(A_{4}\right)$ below;

$\left(A_{1}\right) f_{i}(x, x)=0$, for all $x \in C$, for $i=1,2,3 \ldots k$

$\left(A_{2}\right) f_{i}$ is monotone, i.e., $f_{i}(x, y)+f_{i}(y, x) \leq 0$, for each $i \in\{1,2,3, \ldots, k\}$ and $x, y \in C$

$\left(A_{3}\right)$ for all $x, y, z \in C$, we have $\limsup _{t \rightarrow \infty} f_{i}(t z+(1-t) x, y) \leq f_{i}(x, y)$

$\left(A_{4}\right)$ for all $x \in C, f_{i}(x,$.$) is convex and lower semi-continuous \forall i \in\{1,2,3, \ldots, k\}$.

The mixed equilibrium problem is to find $\widehat{x} \in C$ such that

$$
\sum_{i=1}^{k} f_{i}(\hat{x}, y)+\varphi(y)-\varphi(\hat{x}) \geq 0, \forall y \in C .
$$

The set of solution of (4) is denoted by

$$
\operatorname{GMEP}\left(f_{i}, \varphi\right)=\left\{\hat{x} \in C: \sum_{i=1}^{k} f_{i}(\hat{x}, y)+\varphi(y)-\varphi(\hat{x}) \geq 0, \forall y \in C\right\} .
$$

The generalized mixed equilibrium problem is to find $\widehat{x} \in C$ such that

$$
\sum_{i=1}^{k} f_{i}(\hat{x}, y)+\langle A \hat{x}, y-\hat{x}\rangle+\varphi(y)-\varphi(\hat{x}) \geq 0, \forall y \in C .
$$

The set of solution of (5) is denoted by

$$
\operatorname{GMEP}\left(f_{i}, A, \varphi\right)=\left\{\hat{x} \in C: \sum_{i=1}^{k} f_{i}(\hat{x}, y)+\langle A \hat{x}, y-\hat{x}\rangle+\varphi(y)-\varphi(\hat{x}) \geq 0, \forall y \in C\right\} .
$$

The generalized mixed equilibrium problems are problems that arises in various applications such as in economics, mathematical physics, engineering, and other fields. Moreover, equilibrium problems are closely related with other general problems in nonlinear analysis such as fixed point, game theory, variational inequality, and optimization problems. Some methods have been proposed to solve the equilibrium problem in Hilbert spaces, see for example [2-4] and references contained therein.

In 2007, Tada and Takahashi [5, 6] and Takahashi and Takahashi [7] proved weak and strong convergence theorems for finding a common element of the set of an equilibrium problem and the set of fixed points of a nonexpansive mapping in a Hilbert space. In 2009, Takahashi and Zembayashi [8] introduced two iterative sequences for finding a common element of the set of fixed points of a relatively nonexpansive mapping and the set of solutions of an equilibrium problem in Banach space as follows :

$$
\left\{\begin{array}{l}
x_{0}=x \in C \\
y_{n}=J^{-1}\left(\alpha_{n} J x_{n}+\left(1-\alpha_{n}\right) J S x_{n},\right. \\
u_{n} \in C \text { such that } f\left(u_{n}, y\right)+\frac{1}{r_{n}}\left\langle y-u_{n}, J u_{n}-J y_{n}\right\rangle \geq 0, \forall y \in C \\
H_{n}=\left\{z \in C: \phi\left(z, u_{n}\right) \leq \phi\left(z, x_{n}\right)\right\} \\
W_{n}=\left\{z \in C:\left\langle x_{n}-z, J x-J x_{n}\right\rangle \geq 0\right\} \\
x_{n+1}=\Pi_{H_{n} \cap W_{n}} x, n \geq 0
\end{array}\right.
$$




$$
\left\{\begin{array}{l}
x_{n} \in C \text { such that } f\left(x_{n}, y\right)+\frac{1}{r_{n}}\left\langle y-x_{n}, J x_{n}-J u_{n}\right\rangle \geq 0, \forall y \in C \\
u_{n+1}=J^{-1}\left(\alpha_{n} J x_{n}+\left(1-\alpha_{n}\right) J S x_{n}, \forall n \geq 0\right.
\end{array}\right.
$$

where $J$ is the normalized duality mapping on $E,\left\{\alpha_{n}\right\} \subset[0,1]$ satisfies $\liminf _{n \rightarrow \infty} \alpha_{n}\left(1-\alpha_{n}\right)>$ 0 and $\left\{r_{n}\right\} \subset[a, \infty]$ for some $a>0$. They proved strong convergence of the scheme (6) to a common element of the set of fixed points of relatively nonexpansive mapping and the set of solution of an equilibrium problem in a Banach space. Moreover, they proved weak convergence using scheme (7). In 2012, Chang et al. [9] considered the class of uniformly quasi- $\phi$-asymptotically nonexpansive nonself mappings and studied in a uniformly convex and uniformly smooth real Banach space. In 2014, Deng et al. [10] proved strong convergence theorems of the hybrid algorithm for common fixed point problem of finite family of asymptotically nonexpansive mappings and the set of solution of mixed equilibrium problem in uniformly smooth and uniformly convex Banach spaces. In 2016, Ezeora [11] proved strong convergence theorems for a common element of the set of solution of generalized mixed equilibrium problem and the set of common fixed points of a finite family of multivalued strictly pseudocontractive mappings in real Hilbert spaces.

In this paper, motivated and inspired by the results mentioned above, we prove strong and weak convergence theorems for finding a common element of the set of fixed point of a quasi- $\phi$-asymptotically nonexpansive multivalued mapping and the sets of solutions of generalized mixed equilibrium problem in Banach space. Our results generalized and improve recent results announced by many authors.

\section{Preliminaries}

Throughout this paper, we denoted by $\mathbb{N}$ and $\mathbb{R}$ the sets of positive integer and real numbers, respectively. Let $E$ be a Banach space and $E^{*}$ be the dual of $E$; we denote the strong convergence and the weak convergence of a sequence $\left\{x_{n}\right\}$ to $x$ in $E$ by $x_{n} \longrightarrow x$ and $x_{n} \rightarrow x$ respectively. We also denote the weak* convergence of a sequence $\left\{x_{n}^{*}\right\}$ to $x^{*}$ in $E^{*}$ by $x_{n}^{*} \rightarrow x^{*}$; for all $x \in E$ and $x^{*} \in E^{*}$, we denote the value of $x^{*}$ at $x$ by $\left\langle x, x^{*}\right\rangle$, which is called duality pairing. The normalized duality mapping $J$ on $E$ is defined by

$$
J(x)=\left\{x^{*} \in E^{*}:\left\langle x, x^{*}\right\rangle=\|x\|^{2}=\left\|x^{*}\right\|^{2}\right\} .
$$

for every $x \in E$. A Banach space $E$ is said to be strictly convex if $\frac{\|x+y\|}{2}<1$ for all $x, y \in E$ with $\|x\|=\|y\|=1$ and $x \neq y$. The space $E$ is also said to be uniformly convex if for each $\varepsilon \in(0,2]$, there exists $\delta>0$ such that $\frac{\|x+y\|}{2} \leq 1-\delta$ for all $x, y \in E$ with $\|x\|=\|y\|=1$ and $\|x-y\| \geq \varepsilon$. A Banach space is said to have Kadec-Klee property, if for $x_{n} \rightarrow x$ and $\left\|x_{n}\right\| \longrightarrow\|x\|$ imply $x_{n} \longrightarrow x$. Every Hilbert space and uniformly convex Banach space has Kadec-Klee property. The space $E$ is said to be smooth if the $\lim _{n \rightarrow 0} \frac{\|x+t y\|-\|x\|}{t}$ exists for all $x, y \in S(E)=\{z \in E:\|z\|=1\}$. It is also said to be uniformly smooth if the limit exists uniformly in $x, y \in S(E)$. It is known that if $E$ is smooth, strictly convex, and reflexive, then the duality mapping $J$ is single-valued, oneto-one, and onto. The duality mapping $J$ is said to be weakly sequentially continuous if for any sequence $\left\{x_{n}\right\}$ in $E, x_{n} \rightarrow x$ implies $J x_{n} \rightarrow J x$, see [12].

Let $E$ be a smooth, strictly convex, and reflexive Banach space and $C$ be a nonempty closed convex subset of $E$. Throughout this paper, we denote by $\phi$ the function defined by $\phi(y, x)=\|y\|^{2}-2\langle y, J x\rangle+\|x\|^{2}, \forall x, y \in E$. It is clear from the definition of the function 
$\phi$ that for all $x, y, z \in E$, we have $(\|y\|-\|x\|)^{2} \leq \phi(x, y) \leq(\|y\|+\|x\|)^{2}$ and $\phi(x, y)=$ $\phi(x, z)+\phi(z, y)+\langle x-z, J z-J y\rangle$. Following Albert [13], the generalized projection $\Pi_{C}$ from $E$ onto $C$ is defined by $\Pi_{C}(x)=\operatorname{argmin} \phi(y, x), \forall x \in E$ and $y \in C$. If $E$ is a Hilbert space $H$, then $\phi(y, x)=\|y-x\|^{2}$ and $\Pi_{C}$ become the metric projection of $H$ onto $C$.

The following lemmas for generalized projections are well known.

Lemma 1 (see [13]) Let $C$ be a nonempty closed convex subset of a smooth, strictly con$v e x$, and reflexive Banach space $E$. Then, $\phi\left(x, \Pi_{C} y\right)+\phi\left(\Pi_{C} y, y\right) \leq \phi(x, y), \forall x \in C$ and $y \in E$.

Lemma 2 (see $[13,14])$ Let $C$ be a nonempty closed convex subset of a smooth, strictly convex, and reflexive Banach space. Let $x \in E$ and $z \in C$. Then, $z=\Pi_{C} x \Longleftrightarrow$ $\langle y-z, J x-J y\rangle \leq 0, \forall y \in C$.

A mapping $T: C \longrightarrow C$ is called nonexpansive if $\|T x-T y\| \leq\|x-y\|, \forall x, y \in C$. We denote by $F(T)$ the set of fixed points of $T$ see [15]. A point $p \in C$ is said to be an asymptotic fixed point of $T$ if there exists a sequence $\left\{x_{n}\right\}$ in $C$ which converges weakly to $p$ and $\lim _{n \rightarrow \infty}\left\|x_{n}-T x_{n}\right\|=0$. We denote the set of all asymptotic fixed points of $T$ by $\hat{F}(T)$. Following Matsushita and Takahashi [16-18], a mapping $T$ of $C$ into itself is said to be relatively nonexpansive if the following conditions are satisfied:

(i) $F(T) \neq \emptyset$

(ii) $\phi(p, T x) \leq \phi(p, x), \forall x \in C, p \in F(T)$ and

(iii) $F(T)=\hat{F}(T)$.

Let $C$ be a nonempty closed convex subset of a Banach space $E$. Let $\hat{C} B(C)$ be the families of nonempty, closed, and bounded subsets of $C$

Definition 1 A multivalued mapping $T: C \longrightarrow \hat{C} B(C)$ is said to be relatively nonexpansive if

(i) $F(T) \neq \varnothing$

(ii) $\phi(p, \omega) \leq \phi(p, x), \forall x \in C, \omega \in T x, p \in F(T)$ and

(iii) $F(T)=\hat{F}(T)$.

A multivalued mapping $T: C \longrightarrow \hat{C} B(C)$ is said to be closed if for any sequence $\left\{x_{n}\right\} \subset C$ with $x_{n} \longrightarrow x$ and $\omega_{n} \in T\left(x_{n}\right)$ with $\omega_{n} \longrightarrow y$ then $y \in T x$.

Definition 2 A multivalued mapping $T: C \longrightarrow \hat{C} B(C)$ is said to be quasi- $\phi$ - nonexpansive if

(i) $F(T) \neq \emptyset$ and

(ii) $\phi(p, \omega) \leq \phi(p, x), \forall x \in C, \omega \in T x, p \in F(T)$.

A multivalued mapping $T: C \longrightarrow \hat{C} B(C)$ is a said be quasi- $\phi$-asymptotically nonexpansive if

(i) $F(T) \neq \emptyset$

(ii) There exists a real sequence $\left\{k_{n}\right\} \subset[1, \infty)$ with $k_{n} \rightarrow 1$ such that

$\phi\left(p, \omega_{n}\right) \leq k_{n} \phi(p, x), \forall n \geq 1, x \in C, \omega_{n} \in T^{n} x, p \in F(T)$. 
Lemma 3 (see [14]) Let E be a smooth and uniformly convex Banach space and let $\left\{x_{n}\right\}$ and $\left\{y_{n}\right\}$ be sequences in $E$ such that either $\left\{x_{n}\right\}$ or $\left\{y_{n}\right\}$ is bounded. If $\lim _{n \rightarrow \infty} \phi\left(x_{n}, y_{n}\right)=0$, then $\lim _{n \rightarrow \infty}\left\|x_{n}-y_{n}\right\|=0$.

Lemma 4 (see [19]) Let E be a uniformly convex Banach space. For arbitrary $r>0$, let $B_{r}(0):=\{\|x \in E:\| x \| \leq r\}$. Then, for any given sequence $\left\{x_{n}\right\}_{n=1}^{\infty} \subset B_{r}(0)$ and for any given sequence $\{\lambda\}_{n=1}^{\infty}$ of positive numbers such that $\sum_{n=1}^{\infty} \lambda_{n}=1$, there exists a continuous strictly increasing convex function

$$
g:[0,2 r] \longrightarrow \mathbb{R}, g(0)=0
$$

such that for any positive integers $i, j$ with $i<j$, the following inequality holds:

$$
\left\|\sum_{n=1}^{\infty} \lambda_{n} x_{n}\right\|^{2} \leq \sum_{n=1}^{\infty} \lambda_{n}\left\|x_{n}\right\|^{2}-\lambda_{i} \lambda_{j} g\left(\left\|x_{i}-x_{j}\right\|\right) .
$$

Lemma 5 (see [20]) Let E be a smooth and uniformly convex Banach space and let $r>0$. Then, there exists a strictly increasing continuous and convex function $g:[0,2 r] \longrightarrow \mathbb{R}$ such that $g(0)=0$ and $g_{1}(\|x-y\|) \leq \phi(x, y)$, for all $x, y \in B_{r}$.

Lemma 6 (see [21]) Let $\left\{a_{n}\right\},\left\{b_{n}\right\}$, and $\left\{c_{n}\right\}$ be sequences of nonnegative real numbers satisfying $a_{n+1} \leq\left(1+c_{n}\right) a_{n}+b_{n}$, for all $n \in \mathbb{N}$, where $\sum_{n=1}^{\infty} b_{n}<\infty$ and $\sum_{n=1}^{\infty} c_{n}<\infty$. Then,

(i) $\lim _{n \rightarrow \infty} a_{n}$ exists.

(ii)if $\liminf _{n \rightarrow \infty} a_{n}=0$, then $\lim _{n \rightarrow \infty} a_{n}=0$.

Lemma 7 (see [22]) Let E be a smooth, strictly convex, and reflexive Banach space and $C$ be a nonempty closed convex subset of $E$. Let $B: C \longrightarrow E^{*}$ be a continuous and monotone mapping, $\zeta: C \longrightarrow \mathbb{R}$ be a lower semi-continuous and convex function, and $h: C \times C \longrightarrow$ $\mathbb{R}$ be a bifunction satisfying the conditions $(A 1)-(A 4)$. Let $r>0$ be any given number and $u \in E$ be any given point. Then, the following hold:

(1) There exists $z \in C$ such that

$$
h(z, v)+\zeta(v)-\zeta(z)+\langle v-z, B z\rangle+\frac{1}{r}\langle v-z, J z-J u\rangle \geq 0, \forall v \in C .
$$

(2) If we define a mapping $A_{r}: E \longrightarrow C$ by

$A_{r}(u)=\left\{z \in C: h(z, v)+\zeta(v)-\zeta(z)+\langle v-z, B z\rangle+\frac{1}{r}\langle v-z, J z-J u\rangle \geq 0, \forall v \in C\right\}, u \in E$,

the mapping $A_{r}$ has the following properties:

(a) $A_{r}$ is single-valued;

(b) $F\left(A_{r}\right)=\operatorname{GMEP}(h, A, \zeta)=\hat{F}\left(A_{r}\right)$

(c) $\operatorname{GMEP}(h, A, \zeta)$ is a closed convex subset of $C$;

(d) $\phi\left(q, A_{r} u\right)+\phi\left(A_{r} u, u\right) \leq \phi(q, u), \forall q \in F\left(A_{r}\right), u \in E$.

where $\hat{F}\left(A_{r}\right)$ denotes the set of asymptotic fixed points of $A_{r}$, i.e.,

$$
\hat{F}\left(A_{r}\right):=\left\{x \in C: \exists\left\{x_{n}\right\} \subset C \text {, s.t } x_{n} \rightarrow x,\left\|x_{n}-A_{r} x_{n}\right\| \longrightarrow 0(n \longrightarrow \infty)\right\}
$$

\section{Strong convergence theorem}

In this section, we prove a strong convergence theorem for finding a common element of 
the set of solutions of generalized mixed equilibrium problems and the set of fixed point of quasi- $\phi$-asymptotically nonexpansive multivalued mapping in Banach space.

Theorem 1 Let E be a uniformly smooth and uniformly convex Banach space, and Let $C$ be a nonempty closed convex subset of $E$ and $\hat{C} B(C)$ be the family of nonempty, closed, and bounded subsets of $C$. Let $f_{i}: C \times C \longrightarrow \mathbb{R}, i=1,2,3, \ldots k$ be bi functions which satisfy the conditions $\left(A_{1}\right)-\left(A_{4}\right), A: C \longrightarrow E^{*}$ be a nonlinear mapping, and $\varphi: C \longrightarrow$ $\mathbb{R} \cup\{\infty\}$ be a proper, convex, and lower semi-continuous function. Let $T_{i}, i=1,2,3, \ldots N$ be a quasi- $\phi$-asymptotically nonexpansive multivalued mapping from $C$ into $\hat{C} B(C)$ such that $F(T) \cap \operatorname{GMEP}(f, A, \varphi) \neq \emptyset$. Let $\left\{x_{n}\right\}$ be a sequence generated by

$$
\left\{\begin{array}{l}
x_{0}=x \in C \\
y_{n}=J^{-1}\left(\alpha_{0, n} J x_{n}+\sum_{i=1}^{N} \alpha_{i, n} J w_{i, n}\right), \\
u_{n} \in C \text { such that } \sum_{i=1}^{K} f_{i}\left(u_{n}, y\right)+\varphi(y)-\varphi\left(u_{n}\right)+\left\langle y-u_{n}, A u_{n}\right\rangle \\
+\frac{1}{r_{n}}\left\langle y-u_{n}, J u_{n}-J y_{n}\right\rangle \geq 0, \forall y \in C \\
M_{n}=\left\{z \in C: \phi\left(z, u_{n}\right) \leq k_{n}^{2} \phi\left(z, x_{n}\right)\right\} \\
W_{n}=\left\{z \in C:\left\langle x_{n}-z, J x-J x_{n}\right\rangle \geq 0\right\} \\
x_{n+1}=\Pi_{M_{n} \cap W_{n}} x, \forall n \geq 0,
\end{array}\right.
$$

where $J$ is the normalized duality mapping of $E,\left\{\alpha_{i, n}\right\} \subset[0,1]$ satisfies $\liminf _{n \rightarrow \infty} \alpha_{0, n} \alpha_{i, n}>0$, $\sum_{i=0}^{N} \alpha_{i, n}=1$ and $w_{i, n} \in T_{i}^{n} x_{n}, \forall_{i}=1,2,3, \ldots N .\left\{r_{n}\right\} \subset[a, \infty]$, some $a>0$. Then, $\left\{x_{n}\right\}$ converges strongly to $\Pi_{F(T) \cap G M E P(f, A, \varphi)}$ x, where $\Pi_{F(T) \cap G M E P(f, A, \varphi)}$ is the generalized projection of $E$ onto $F(T) \cap \operatorname{GMEP}(f, A, \varphi)$,

Proof Let two functions $\tau: C \times C \longrightarrow \mathbb{R}$ and $T_{r}: E \longrightarrow C$ be defined by

$$
\tau(x, y)=\sum_{i=1}^{k} f_{i}(x, y)+\langle A x, y-x\rangle+\varphi(y)-\varphi(x), \forall x, y \in C
$$

and

$$
T_{r}(x)=\left\{u \in C: \tau(u, y)+\frac{1}{r}\langle y-u, J u-J x\rangle \geq 0, \forall y \in C, \forall x \in E\right.
$$

respectively. Now, the function $\tau$ satisfies conditions $(A 1)-(A 4)$ and $T_{r}$ has the properties $(a)-(d)$. Therefore, iterative sequence (8) can be rewritten as

$$
\left\{\begin{array}{l}
x_{0}=x \in C \\
y_{n}=J^{-1}\left(\alpha_{0, n} J x_{n}+\sum_{i=1}^{N} \alpha_{i, n} J w_{i, n}\right), w_{i, n} \in T_{i}^{n} x_{n}, n \geq 1 \\
u_{n} \in C \text { such that } \tau\left(u_{n}, y\right)+\frac{1}{r_{n}}\left\langle y-u_{n}, J u_{n}-J y_{n}\right\rangle \geq 0, \forall y \in C \\
M_{n}=\left\{z \in C: \phi\left(z, u_{n}\right) \leq k_{n}^{2} \phi\left(z, x_{n}\right)\right\} \\
W_{n}=\left\{z \in C:\left\langle x_{n}-z, J x-J x_{n}\right\rangle \geq 0\right\} \\
x_{n+1}=\Pi_{M_{n} \cap W_{n} x}, N \in \mathbb{N} .
\end{array}\right.
$$

We first show that $M_{n} \cap W_{n}$ is closed and convex, and it is obvious that $M_{n}$ is closed and convex since $\phi\left(z, u_{n}\right) \leq k_{n}^{2} \phi\left(z, x_{n}\right) \Longleftrightarrow\left(1-k_{n}^{2}\right)\|z\|^{2}-2\left(1-k_{n}^{2}\right)\left\langle z, J u_{n}\right\rangle+$ $2 k_{n}^{2}\left\langle z, J x_{n}-J u_{n}\right\rangle \leq k_{n}^{2}\left\|x_{n}\right\|^{2}-\left\|u_{n}\right\|^{2}$.

Thus, $M_{n} \cap W_{n}$ is a closed and convex subset of $E$ for all $n \in \mathbb{N} \cup\{0\}$, so that $\left\{x_{n}\right\}$ is well defined. 
Let $u \in F(T) \cap \operatorname{GMEP}(f, A, \varphi)$, putting $u_{n}=\omega_{n} \in T_{r_{n}} y_{n}$ for all $n \in \mathbb{N} \cup\{0\}$, and since $T_{r_{n}}$ are quasi- $\phi$-asymptotically nonexpansive multivalued, we have

$$
\begin{aligned}
\phi\left(u, u_{n}\right) & =\phi\left(u, \omega_{n}\right) \\
& \leq k_{n} \phi\left(u, y_{n}\right) \\
& =k_{n}\left[\phi\left(u, J^{-1}\left(\alpha_{0, n} J x_{n}+\sum_{i=1}^{N} \alpha_{i, n} J w_{i, n}\right)\right)\right], \\
& =k_{n}\left[\|u\|^{2}-2\left\langle u, \alpha_{i, 0} J x_{n}+\sum_{i=1}^{N} \alpha_{i, n} J w_{i, n}\right\rangle+\left\|\alpha_{0, n} J x_{n}+\sum_{i=1}^{N} \alpha_{i, n} J w_{i, n}\right\|^{2}\right. \\
& \leq k_{n}\left[\|u\|^{2}-2\left(\left\langle u, \alpha_{0, n} J x_{n}\right\rangle+\left\langle u, \sum_{i=1}^{N} \alpha_{i, n} J w_{i, n}\right\rangle\right)+\alpha_{0, n}\left\|J x_{n}\right\|^{2}+\sum_{i=1}^{N} \alpha_{i, n}\left\|J w_{i, n}\right\|^{2}\right] \\
& =k_{n}\left[\alpha_{0, n}\left(\|u\|^{2}-2\left\langle u, J x_{n}\right\rangle+\left\|x_{n}\right\|^{2}\right)+\sum_{i=1}^{N} \alpha_{i, n}\left(\|u\|^{2}-2\left\langle u, J w_{i, n}\right\rangle+\left\|w_{i, n}\right\|^{2}\right)\right] \\
& =k_{n}\left[\alpha_{0, n} \phi\left(u, x_{n}\right)+\sum_{i=1}^{N} \alpha_{i, n} \phi\left(u, w_{i, n}\right)\right] \\
& \leq k_{n}\left[\alpha_{0, n} \phi\left(u, x_{n}\right)+k_{n} \sum_{i=1}^{N} \alpha_{i, n} \phi\left(u, x_{n}\right)\right] \\
& \leq k_{n}\left[k_{n} \alpha_{0, n} \phi\left(u, x_{n}\right)+k_{n} \sum_{i=1}^{N} \alpha_{i, n} \phi\left(u, x_{n}\right)\right] \\
& =k_{n}\left[\left(\alpha_{0, n}+\sum_{i=1}^{N} \alpha_{i, n}\right) k_{n} \phi\left(u, x_{n}\right)\right] \\
& k_{n}^{2} \phi\left(u, x_{n}\right) .
\end{aligned}
$$

Hence, we have $u \in M_{n}$. This implies that $F(T) \cap \operatorname{GMEP}(f, A, \varphi) \subset M_{n}, \forall n \in \mathbb{N} \cup\{0\}$. Next, we show by induction that $F(T) \cap \operatorname{GMEP}(f, A, \varphi) \subset M_{n} \cap W_{n}, \forall n \in \mathbb{N} \cup\{0\}$.

From $W_{0}=C$, we have

$$
F(T) \cap \operatorname{GMEP}(f, A, \varphi) \subset M_{0} \cap W_{0}, .
$$

Suppose that $F(T) \cap \operatorname{GMEP}(f, A, \varphi) \subset M_{k} \cap W_{k}$, for some $k \in \mathbb{N} \cup\{0\}$. Then, there exists $x_{k+1} \in M_{k} \cap W_{k}$ such that $x_{k+1}=\Pi_{M_{k} \cap W_{k} x}$

From the definition of $x_{k+1}$, we have for all $z \in M_{k} \cap W_{k}$,

$$
\left\langle x_{k+1}-z, J x-J x_{k+1}\right\rangle \geq 0
$$

Since $F(T) \cap G M E P(f, A, \varphi) \subset M_{k} \cap W_{k}$, we have

$$
\left\langle x_{k+1}-z, J x-J x_{k+1}\right\rangle \geq 0
$$

$\forall z \in F(T) \cap \operatorname{GMEP}(f, A, \varphi)$ and so $z \in W_{k+1}$. Thus, $F(T) \cap \operatorname{GMEP}(f, A, \varphi) \subset W_{k+1}$. Therefore, we have $F(T) \cap \operatorname{GMEP}(f, A, \varphi) \subset M_{k+1} \cap W_{k+1}$. Therefore, we obtain 


$$
F(T) \cap \operatorname{GMEP}(f, A, \varphi) \subset M_{n} \cap W_{n}, \forall n \in \mathbb{N} \cup\{0\} .
$$

From the definition of $W_{n}$, we have $x_{n}=\Pi_{W_{n}} x$; using this and Lemma 1, we have

$$
\begin{aligned}
\phi\left(x_{n}, x\right) & =\phi\left(\Pi_{W_{n}} x, x\right) \leq \phi(u, x)-\phi\left(u, \Pi_{W_{n}} x,\right) \\
& \leq \phi(u, x)
\end{aligned}
$$

for all $u \in F(T) \cap \operatorname{GMEP}(f, A, \varphi) \subset W_{n}$. Therefore, $\phi\left(x_{n}, x\right)$ is bounded, and consequently $\left\{x_{n}\right\}$ and $\left\{T_{i}^{n} x_{n}\right\}$ are bounded.

Since $x_{n+1}=\Pi_{M_{n} \cap W_{n}} x \in M_{n} \cap W_{n} \subset W_{n}$ and $x_{n}=\Pi_{W_{n}} x$, we have

$$
\phi\left(x_{n}, x\right) \leq \phi\left(x_{n+1}, x\right), \forall n \in \mathbb{N} \cup\{0\} .
$$

Thus, $\left\{\phi\left(x_{n}, x\right)\right\}$ is nondecreasing. Using (10) and (11), we have the limit of $\left\{\phi\left(x_{n}, x\right)\right\}$ exists.

From $x_{n}=\Pi_{W_{n}} x$ and Lemma 1, we also have

$$
\phi\left(x_{n+1}, x_{n}\right)=\phi\left(x_{n+1}, \Pi_{W_{n}} x,\right) \leq \phi\left(x_{n+1}, x\right)-\phi\left(\Pi_{W_{n}} x, x\right)=\phi\left(x_{n+1}, x\right)-\phi\left(x_{n}, x\right)
$$

for all $n \in \mathbb{N} \cup\{0\}$. This means that $\lim _{n \rightarrow \infty} \phi\left(x_{n+1}, x_{n}\right)=0$.

From $x_{n+1}=\Pi_{M_{n} \cap W_{n}} x \in M_{n}$ and the definition of $M_{n}$, we have

$$
\phi\left(x_{n+1}, u_{n}\right) \leq k_{n}^{2} \phi\left(x_{n+1}, x_{n}\right), \forall n \in \mathbb{N} \cup\{0\} .
$$

Therefore, we have

$\lim _{n \rightarrow \infty} \phi\left(x_{n+1}, u_{n}\right)=0$. As $E$ is uniformly convex and smooth, we have from Lemma 3 that

$$
\lim _{n \rightarrow \infty}\left\|x_{n+1}-x_{n}\right\|=\lim _{n \rightarrow \infty}\left\|x_{n+1}-u_{n}\right\|=0
$$

From which, we have

$$
\lim _{n \rightarrow \infty}\left\|x_{n}-u_{n}\right\|=0
$$

Since $J$ is uniformly norm-to-norm continuous on bounded sets, we have

$$
\lim _{n \rightarrow \infty}\left\|J x_{n}-J u_{n}\right\|=0
$$

Let $r=\sup _{n \in \mathbb{N}}\left\{\left\|x_{n}\right\|,\left\|T_{i}^{n} x_{n}\right\|\right\}$. Since $E$ is a uniformly smooth Banach space, we know that $E^{*}$ is a uniformly convex Banach space. So, for $u \in F(T) \cap \operatorname{GMEP}(f, A, \varphi)$, putting $u_{n}=\omega_{n}=T_{r_{n}} y_{n}$ and using Lemma 4 , we have : 


$$
\begin{aligned}
\phi\left(u, u_{n}\right)= & \phi\left(u, \omega_{n}\right) \\
\leq & k_{n} \phi\left(u, y_{n}\right) \\
= & k_{n}\left[\phi\left(u, J^{-1}\left(\alpha_{0, n} J x_{n}+\sum_{i=1}^{N} \alpha_{i, n} J w_{i, n}\right)\right)\right], \\
= & k_{n}\left[\|u\|_{2}-2\left\langle u, \alpha_{i, 0} J x_{n}+\sum_{i=1}^{N} \alpha_{i, n} J w_{i, n}\right\rangle+\left\|\alpha_{0, n} J x_{n}+\sum_{i=1}^{N} \alpha_{i, n} J w_{i, n}\right\|^{2}\right] \\
\leq & k_{n}\left[\|u\|^{2}-2\left(\left\langle u, \alpha_{0, n} J x_{n}\right\rangle+\left\langle u, \sum_{i=1}^{N} \alpha_{i, n} J w_{i, n}\right\rangle\right)+\alpha_{0, n}\left\|J x_{n}\right\|^{2}+\sum_{i=1}^{N} \alpha_{i, n}\left\|J w_{i, n}\right\|^{2}\right. \\
& \left.-\alpha_{0, n} \alpha_{i, n} g\left(\left\|J x_{n}-J w_{i, n}\right\|\right)\right] \\
= & k_{n}\left[\alpha_{0, n}\left[\|u\|^{2}-2\left\langle u, J x_{n}\right\rangle+\left\|x_{n}\right\|^{2}\right]+\sum_{i=1}^{N} \alpha_{i, n}\left[\|u\|^{2}-2\left\langle u, J w_{i, n}\right\rangle+\left\|w_{i, n}\right\|^{2}\right]\right. \\
& \left.-\alpha_{0, n} \alpha_{i, n} g\left(\left\|J x_{n}-J w_{i, n}\right\|\right)\right] \\
= & k_{n}\left[\alpha_{0, n} \phi\left(u, x_{n}\right)+\sum_{i=1}^{N} \alpha_{i, n} \phi\left(u, w_{i, n}\right)-\alpha_{0, n} \alpha_{i, n} g\left(\left\|J x_{n}-J w_{i, n}\right\|\right)\right] \\
\leq & k_{n}\left[\alpha_{0, n} \phi\left(u, x_{n}\right)+\sum_{i=1}^{N} k_{n} \alpha_{i, n} \phi\left(u, x_{n}\right)-\alpha_{0, n} \alpha_{i, n} g\left(\left\|J x_{n}-J w_{i, n}\right\|\right)\right] \\
\leq & k_{n}\left[k_{n} \alpha_{0, n} \phi\left(u, x_{n}\right)+k_{n} \sum_{i=1}^{N} \alpha_{i, n} \phi\left(u, x_{n}\right)-\alpha_{0, n} \alpha_{i, n} g\left(\left\|J x_{n}-J w_{i, n}\right\|\right)\right] \\
= & k_{n}\left[k_{n} \phi\left(u, x_{n}\right)-\alpha_{0, n} \alpha_{i, n} g\left(\left\|J x_{n}-J w_{i, n}\right\|\right)\right] \\
(12) & k_{n} \alpha_{0, n} \alpha_{i, n} g\left(\left\|J x_{n}-J w_{i, n}\right\|\right) . \\
& \\
& \\
&
\end{aligned}
$$

Therefore, from (12), we have

$k_{n} \alpha_{0, n} \alpha_{i, n} g\left(\left\|J x_{n}-J w_{i, n}\right\|\right) \leq k_{n}^{2} \phi\left(u, x_{n}\right)-\phi\left(u, u_{n}\right), \forall n \in \mathbb{N} \cup\{0\}$.

But

$$
\begin{aligned}
& k_{n}^{2} \phi\left(u, x_{n}\right)-\phi\left(u, u_{n}\right)=k_{n}^{2}\left[\|u\|^{2}-2\left\langle u, J x_{n}\right\rangle+\left\|x_{n}\right\|^{2}\right]-\left[\|u\|^{2}-2\left\langle u, J u_{n}\right\rangle+\left\|u_{n}\right\|^{2}\right] \\
&=\left(k_{n}^{2}-1\right)\|u\|^{2}-2\left(k_{n}^{2}-1\right)\left\langle u, J u_{n}\right\rangle-2 k_{n}^{2}\left\langle u, J x_{n}-J u_{n}\right\rangle+k_{n}^{2}\left\|x_{n}\right\|^{2}-\left\|u_{n}\right\|^{2} \\
&=\left(k_{n}^{2}-1\right)\|u\|^{2}-2\left(k_{n}^{2}-1\right)\left\langle u, J u_{n}\right\rangle-2 k_{n}^{2}\left\langle u, J x_{n}-J u_{n}\right\rangle+\left(k_{n}^{2}-1\right)\left\|x_{n}\right\|^{2} \\
&+\left\|x_{n}\right\|^{2}-\left\|u_{n}\right\|^{2} \\
& \leq\left|\left(k_{n}^{2}-1\right)\|u\|^{2}\right|+\left|2\left(k_{n}^{2}-1\right)\left\langle u, J u_{n}\right\rangle\right|+\left|2 k_{n}^{2}\left\langle u, J x_{n}-J u_{n}\right\rangle\right|+\left|\left(k_{n}^{2}-1\right)\left\|x_{n}\right\|^{2}\right| \\
&+\left|\left\|x_{n}\right\|^{2}-\left\|u_{n}\right\|^{2}\right| \\
& \leq\left(k_{n}^{2}-1\right)\|u\|^{2}+2\left(k_{n}^{2}-1\right)\|u\|\left\|J u_{n}\right\|+2 k_{n}^{2}\|u\|\left\|J x_{n}-J u_{n}\right\|+\left(k_{n}^{2}-1\right)\left\|x_{n}\right\|^{2} \\
&+\left(\left\|x_{n}-u_{n}\right\|\right)\left(\left\|x_{n}\right\|+\left\|u_{n}\right\|\right)
\end{aligned}
$$

Hence,

$$
\lim _{n \rightarrow \infty}\left(k_{n}^{2} \phi\left(u, x_{n}\right)-\phi\left(u, u_{n}\right)\right)=0
$$


Since $\liminf _{n \rightarrow \infty} \alpha_{0, n} \alpha_{i, n}>0$, we have

$$
\lim _{n \rightarrow \infty} g\left(\left\|J x_{n}-J w_{i, n}\right\|\right)=0 .
$$

From the property $g$, we have $\lim _{n \rightarrow \infty}\left\|J x_{n}-J w_{i, n}\right\|=0$.

Since $J^{-1}$ is uniformly norm-to-norm continuous on bounded sets, we have

$$
\lim _{n \rightarrow \infty}\left\|x_{n}-w_{i, n}\right\|=0 .
$$

Since $\left\{x_{n}\right\}$ is bounded, there exists a subsequence $\left\{x_{n_{k}}\right\}$ of $\left\{x_{n}\right\}$ such that $x_{n_{k}} \rightarrow \hat{x}$, for some $\hat{x} \in E$. Since $T$ is quasi- $\phi$-asymptotically nonexpansive multivalued mapping and $E$ is a reflexive space, then we have $\hat{x} \in F\left(T_{i}\right)$.

Next, we show that $\hat{x} \in \operatorname{GMEP}(f, A, \varphi)$. From $u_{n}=T_{r_{n}} y_{n}$ Lemma $7(d)$ and (13), we have

$$
\begin{aligned}
\phi\left(u_{n}, y_{n}\right) & =\phi\left(T_{r_{n}} y_{n}, y_{n}\right) \\
& \leq \phi\left(u, y_{n}\right)-\phi\left(u, T_{r_{n}} y_{n}\right) \\
& \leq k_{n}^{2}\left(u, x_{n}\right)-\phi\left(u, T_{r_{n}} y_{n}\right) \\
& =k_{n}^{2} \phi\left(u, x_{n}\right)-\phi\left(u, u_{n}\right)
\end{aligned}
$$

Thus, $\lim _{n \rightarrow \infty} \phi\left(u_{n}, y_{n}\right)=0$.

Since $\stackrel{n \rightarrow \infty}{E}$ is uniformly convex and smooth, we have from Lemma 3 that

$$
\lim _{n \rightarrow \infty}\left\|u_{n}-y_{n}\right\|=0 \text {. }
$$

From $x_{n_{k}} \rightarrow \hat{x},\left\|x_{n}-u_{n}\right\| \longrightarrow 0$ and (14), we have $y_{n_{k}} \rightarrow \hat{x}$ and $u_{n_{k}} \rightarrow \hat{x}$.

As $J$ is uniformly norm-to-norm continuous on bounded sets and (14), we have $\lim _{n \rightarrow \infty}\left\|J u_{n}-J y_{n}\right\|=0$. From $r_{n} \geq a$, we have

$$
\lim _{n \rightarrow \infty}\left\|\frac{J u_{n}-J y_{n}}{r_{n}}\right\|=0
$$

By $u_{n}=T_{r_{n}} y_{n}$, we have

$$
\tau\left(u_{n}, y\right)+\frac{1}{r_{n}}\left\langle y-u_{n}, J u_{n}-J y_{n}\right\rangle \geq 0, \forall y \in C .
$$

Replacing $n$ by $n_{k}$, we have from $\left(A_{2}\right)$ that

$$
\frac{1}{r_{n_{k}}}\left\langle y-u_{n_{n}}, J u_{n_{k}}-J y_{n_{k}}\right\rangle \geq-\tau\left(u_{n_{k}}, y\right) \geq \tau\left(y, u_{n_{k}}\right), \forall y \in C
$$

Letting $k \longrightarrow \infty$, in (16) and using $\left(A_{4}\right)$, we obtain

$$
\tau(y, \hat{x}) \leq 0, \forall y \in C
$$

For $t$ with $0<t \leq 1$ and $y \in C$, let $y_{t}=t y+(1-t) \hat{x}$. Since $y \in C$ and $\hat{x} \in C$, we have $y_{t} \in C$ and $\tau\left(y_{t}, \hat{x}\right) \leq 0, \forall y \in C$. Now, using $\left(A_{1}\right)$ and $\left(A_{3}\right)$, we have

$$
\begin{aligned}
0 & =\tau\left(y_{t}, y_{t}\right) \\
& \leq t \tau\left(y_{t}, y\right)+(1-t) \tau\left(y_{t}, \hat{x}\right) \\
& \leq t \tau\left(y_{t}, y\right) .
\end{aligned}
$$

Dividing by, $t$ we have

$$
\tau\left(y_{t}, y\right) \geq 0, \forall y \in C
$$


Letting $t \longrightarrow 0$, and using $\left(A_{3}\right)$, we have

$$
\tau(\hat{x}, y) \geq 0, \forall y \in C .
$$

This shows that $\hat{x} \in \operatorname{GMEP}(f, A, \varphi)$.

Let $\omega=\Pi_{F(T) \cap G M E P(f, A, \varphi)} x$, From $x_{n+1}=\Pi_{M_{n} \cap W_{n}} x$ and $\omega \in F(T) \cap \operatorname{GMEP}(f, A, \varphi) \subset$ $M_{n} \cap W_{n}$, we have

$$
\phi\left(x_{n+1}, x\right) \leq \phi(\omega, x) .
$$

Since the norm is weakly lower semi-continuous and $x_{n_{k}} \rightarrow \hat{x}$, we have

$$
\begin{aligned}
\phi(\hat{x}, x) & =\|\hat{x}\|^{2}-2\langle\hat{x}, J x\rangle+\|x\|^{2} \\
& \leq \liminf _{k \rightarrow \infty}\left(\left\|x_{n_{k}}\right\|^{2}-2\left\langle x_{n_{k}}, J x\right\rangle+\|x\|^{2}\right) \\
& =\liminf _{k \rightarrow \infty}\left(x_{n_{k}}, x\right) \\
& \leq \limsup _{k \rightarrow \infty}\left(x_{n_{k}}, x\right) \\
& \leq \phi(\omega, x) .
\end{aligned}
$$

From the definition of $\Pi_{F(T) \cap G M E P(f, A, \varphi)}$, we have $\hat{x}=\omega$. Hence, $\lim _{k \rightarrow \infty} \phi\left(x_{n_{k}}, x\right)=$ $\phi(\omega, x)$, Therefore,

$$
\begin{aligned}
0 & =\lim _{k \rightarrow \infty}\left(\phi\left(x_{n_{k}}, x\right)-\phi(\omega, x)\right) \\
& =\lim _{k \rightarrow \infty}\left(\left\|x_{n_{k}}\right\|^{2}-\|\omega\|^{2}-2\left\langle x_{n_{k}}-\omega, J x\right\rangle\right) \\
& =\lim _{k \rightarrow \infty}\left(\left\|x_{n_{k}}\right\|^{2}-\|\omega\|^{2}\right) .
\end{aligned}
$$

Since $E$ has the Kadec-Klee property, we have that $x_{n_{k}} \longrightarrow \omega=\Pi_{F(T) \cap G M E P(f, A, \varphi)} x$.

Therefore, $\left\{x_{n}\right\}$ converges strongly to $\Pi_{F(T) \cap G M E P(f, A, \varphi) x}$.

\section{Weak convergence theorem}

In this section, we prove a weak convergence theorem for finding a common element of the set of solutions of generalized mixed equilibrium problem and the set of fixed point of quasi- $\phi$-asymptotically nonexpansive multivalued mapping in Banach space. Before proving the Theorem, we need the following proposition.

Proposition 1 Let E be a uniformly smooth and uniformly convex Banach space and let $C$ be a nonempty closed convex subset of $E$ and $\hat{C} B(C)$ be the family of nonempty, closed, and bounded subsets of $C$. Let $f_{i}: C \times C \longrightarrow \mathbb{R},(i=1,2,3, \ldots K \in \mathbb{N})$ be bifunctions satisfying $\left(A_{1}\right)-\left(A_{4}\right), A: C \longrightarrow E^{*}$ be a nonlinear mapping and let $\varphi: C \longrightarrow \mathbb{R} \cup\{\infty\}$ be a proper, convex, and lower semi-continuous function. Let $T$ be a quasi- $\phi$-asymptotically nonexpansive multivalued mapping from $C$ into $\hat{C} B(C)$ such that $F(T) \cap \operatorname{GMEP}(f, A, \varphi) \neq$ $\emptyset$. Let $\left\{x_{n}\right\}$ be a sequence generated by $u_{1} \in E$

$$
\left\{\begin{array}{l}
x_{n} \in C \text { such that } \tau\left(x_{n}, y\right)+\frac{1}{r_{n}}\left\langle y-x_{n}, J x_{n}-J u_{n}\right\rangle \geq 0, \forall y \in C \\
u_{n+1}=J^{-1}\left(\alpha_{0, n} J x_{n}+\sum_{i=1}^{N} J w_{i, n}\right), \quad w_{i, n} \in T_{i}^{n} x_{n}, K, N, \in \mathbb{N}
\end{array}\right.
$$

for every $n \in \mathbb{N}$, where $J$ is the normalized duality mapping on $E,\left\{\alpha_{i, n}\right\} \subset[0, \infty)$ satisfying $\liminf _{n \rightarrow \infty} \alpha_{0, n} \alpha_{i, n}>0, \sum_{i=0}^{N} \alpha_{i, n}=1$ and $w_{i, n} \in T_{i}^{n} x_{n}, \forall i=1,2,3, \ldots N \in \mathbb{N}$. 
Let $\left\{r_{n}\right\} \subset(0, \infty)$. Then, $\left\{\Pi_{F(T) \cap G M E P(f, A, \varphi)} x_{n}\right\}$ converges strongly to $z \in F(T) \cap$ $\operatorname{GMEP}(f, A, \varphi)$, where $\Pi_{F(T) \cap G M E P(f, A, \varphi)}$ is generalized projection of $E$ onto $F(T) \cap$ $\operatorname{GMEP}(f, A, \varphi)$.

Proof Let $u \in F(T) \cap \operatorname{GMEP}(f, A, \varphi)$. Putting $x_{n}=\omega_{n} \in T_{r_{n}} u_{n}$ for all $n \in \mathbb{N}$, we know that $T_{r_{n}}$ are quasi- $\phi$-asymptotically nonexpansive multivalued, and we have

$$
\begin{aligned}
\phi & \left(u, x_{n+1}\right) \\
& =\phi\left(u, \omega_{n+1}\right) \\
& \leq k_{n} \phi\left(u, u_{n+1}\right) \\
& =k_{n}\left[\phi\left(u, J^{-1}\left(\alpha_{0, n} J x_{n}+\sum_{i=1}^{N} \alpha_{i, n} J w_{i, n}\right)\right)\right], \quad w_{i, n} \in T_{i}^{n} x_{n}, n \geq 1 \\
& =k_{n}\left[\|u\|^{2}-2\left\langle u, \alpha_{i, 0} J x_{n}+\sum_{i=1}^{N} \alpha_{i, n} J w_{i, n}\right\rangle+\left\|\alpha_{0, n} J x_{n}+\sum_{i=1}^{N} \alpha_{i, n} J w_{i, n}\right\|^{2}\right] \\
& \leq k_{n}\left[\|u\|^{2}-2\left(\left\langle u, \alpha_{0, n} J x_{n}\right\rangle+\left\langle u, \sum_{i=1}^{N} \alpha_{i, n} J w_{i, n}\right\rangle\right)+\alpha_{0, n}\left\|J x_{n}\right\|\left\|^{2}+\sum_{i=1}^{N} \alpha_{i, n}\right\| J w_{i, n} \|^{2}\right] \\
& =k_{n}\left[\alpha_{0, n}\left(\|u\|^{2}-2\left\langle u, J x_{n}\right\rangle+\left\|x_{n}\right\|^{2}\right)+\sum_{i=1}^{N} \alpha_{i, n}\left(\|u\|^{2}-2\left\langle u, J w_{i, n}\right\rangle+\left\|w_{i, n}\right\|^{2}\right)\right] \\
& \leq k_{n}\left[\alpha_{0, n} \phi\left(u, x_{n}\right)+k_{n} \sum_{i=1}^{N} \alpha_{i, n} \phi\left(u, x_{n}\right)\right] \\
& \leq k_{n}\left[k_{n} \alpha_{0, n} \phi\left(u, x_{n}\right)+k_{n} \sum_{i=1}^{N} \alpha_{i, n} \phi\left(u, x_{n}\right)\right] \\
& =k_{n}^{2} \phi\left(u, x_{n}\right) .
\end{aligned}
$$

Thus,

$$
\phi\left(u, x_{n+1}\right) \leq k_{n}^{2} \phi\left(u, x_{n}\right)
$$

Hence, we have

$$
\phi\left(u, x_{n+1}\right) \leq k_{n}^{2} \phi\left(u, x_{n}\right)=\left(1+\left(k_{n}^{2}-1\right)\right) \phi\left(u, x_{n}\right)
$$

By Lemma $6, \sum_{i=1}^{\infty}\left(k_{n}^{2}-1\right)<\infty$, we obtain $\lim _{n \rightarrow \infty} \phi\left(u, x_{n}\right)$ exists. It follows that $\left\{x_{n}\right\}$ and $\left\{w_{i, n}\right\}$ are bounded.

Define $y_{n}=\Pi_{F(T) \cap G M E P(f, A, \varphi)} x_{n}$ for all $n \in \mathbb{N}$. Then, $y_{n} \in F(T) \cap \operatorname{GMEP}(f, A, \varphi)$; therefore, from (17), we have

$$
\phi\left(y_{n}, x_{n+1}\right) \leq k_{n}^{2} \phi\left(y_{n}, x_{n}\right) .
$$

Thus,

$$
\begin{aligned}
\phi\left(y_{n+1}, x_{n+1}\right) & =\phi\left(\Pi_{F(T) \cap G M E P(f, A, \varphi)} x_{n+1}, x_{n+1}\right) \\
& \leq \phi\left(y_{n}, x_{n+1}\right)-\phi\left(y_{n}, \Pi_{\left.F(T) \cap G M E P(f, A, \varphi)^{x_{n+1}}\right)}\right. \\
& =\phi\left(y_{n}, x_{n+1}\right)-\phi\left(y_{n}, y_{n+1}\right) \\
& \leq \phi\left(y_{n}, x_{n+1}\right) .
\end{aligned}
$$


Hence, from (18), we have $\phi\left(y_{n+1}, x_{n+1}\right) \leq k_{n}^{2} \phi\left(y_{n}, x_{n}\right)=\left(1+\left(k_{n}^{2}-1\right)\right) \phi\left(y_{n}, x_{n}\right)$.

By the assumption $\sum_{i=1}^{\infty}\left(k_{n}^{2}-1\right)<\infty$ and using Lemma 6 , we have $\lim _{n \rightarrow \infty} \phi\left(y_{n}, x_{n}\right)$. For $m \in \mathbb{N}$ such that $m>n$, we also have from (18) that

$$
\phi\left(y_{n}, x_{n+m}\right) \leq\left(k_{n}^{2}\right)^{m} \phi\left(y_{n}, x_{n}\right) .
$$

From $y_{n+m}=\Pi_{F(T) \cap G M E P(f, A, \varphi)} x_{n+m}$ and Lemma 1, we have

$$
\begin{aligned}
\phi\left(y_{n}, y_{n+m}\right)+\phi\left(y_{n+m}, x_{n+m}\right) & \leq \phi\left(y_{n}, x_{n+m}\right) \\
& \leq\left(k_{n}^{2}\right)^{m} \phi\left(y_{n}, x_{n}\right)
\end{aligned}
$$

Hence,

$\phi\left(y_{n}, y_{n+m}\right) \leq\left(k_{n}^{2}\right)^{m} \phi\left(y_{n}, x_{n}\right)-\phi\left(y_{n+m}, x_{n+m}\right)$. Let $r=\sup _{n \in \mathbb{N}}\left\|y_{n}\right\| ;$

from Lemma 5 , we have

$$
g\left(\left\|y_{n}-y_{n+m}\right\|\right) \leq \phi\left(y_{n}, y_{n+m}\right) \leq\left(k_{n}^{2}\right)^{m} \phi\left(y_{n}, x_{n}\right)-\phi\left(y_{n+m}, x_{n+m}\right)
$$

Since $\lim _{n \rightarrow \infty} \phi\left(y_{n}, x_{n}\right)$ exists, from the property of $g$, we have that $\left\{y_{n}\right\}$ is Cauchy. Since $F(T) \cap G M E P(f, A, \varphi)$ is closed, $\left\{y_{n}\right\}$ converges strongly to $z \in F(T) \cap \operatorname{GMEP}(f, A, \varphi)$.

Now, we prove the following theorem.

Theorem 2 Let E be a real uniformly smooth and uniformly convex Banach space, let $C$ be a nonempty, closed, and convex subset of $E$, and let $\hat{C} B(C)$ be family of nonempty, closed, and bounded subsets of $C$. Let $f_{i}: C \times C \longrightarrow \mathbb{R},(i=1,2,3, \ldots K)$ be bifunctions satisfying $\left(A_{1}\right)-\left(A_{4}\right), A: C \longrightarrow E^{*}$ be a nonlinear mapping, and $\varphi: C \longrightarrow \mathbb{R} \cup\{\infty\}$ be a proper, convex, and lower semi-continuous function. Let $T$ be a quasi- $\phi$-asymptotically nonexpansive multivalued mapping from $C$ into $\hat{C} B(C)$ such that $F(T) \cap \operatorname{GMEP}(f, A, \varphi) \neq$ $\emptyset$. Let $\left\{x_{n}\right\}$ be a sequence generated by $u_{1} \in E$

$$
\left\{\begin{array}{l}
x_{n} \in C \text { such that } \tau\left(x_{n}, y\right)+\frac{1}{r_{n}}\left\langle y-x_{n}, J x_{n}-J u_{n}\right\rangle \geq 0, \forall y \in C . \\
u_{n+1}=J^{-1}\left(\alpha_{0, n} J x_{n}+\sum_{i=1}^{N} J w_{i, n}\right), \quad w_{i, n} \in T_{i}^{n} x_{n}, K, N, \in \mathbb{N}
\end{array}\right.
$$

for every $n \in \mathbb{N}$, where $J$ is the normalized duality mapping of $E,\left\{\alpha_{i, n}\right\} \subset[0, \infty]$ satisfies $\liminf _{n \rightarrow \infty} \alpha_{0, n} \alpha_{i, n}>0, \sum_{i=0}^{N} \alpha_{i, n}=1$ and $w_{i, n} \in T_{i}^{n} x_{n}, \forall i=1,2,3, \ldots N \in \mathbb{N}$.

Let $\left\{r_{n}\right\} \subset[a, \infty)$ for some $a>0$. If $J$ is weakly sequentially continuous, then $\left\{x_{n}\right\}$ converges weakly to $z \in F(T) \cap \operatorname{GMEP}(f, A, \varphi)$, where $z=\lim _{n \rightarrow \infty} \Pi_{F(T) \cap G M E P(f, A, \varphi)} x_{n}$.

Proof As in the proof of proposition 17, we have that $\left\{x_{n}\right\}$ and $\left\{w_{i, n}\right\}$ and $\left\{T_{i}^{n} x_{n}\right\}$ are bounded sequences. Let $r=\sup _{n \in \mathbb{N}}\left\{\left\|x_{n}\right\|,\left\|w_{i, n}\right\|\right\}$. For $u \in F(T) \cap \operatorname{GMEP}(f, A, \varphi)$, we have

$$
\begin{aligned}
\phi\left(u, x_{n+1}\right) & =\phi\left(u, \omega_{n+1}\right) \\
& \leq k_{n} \phi\left(u, u_{n+1}\right) \\
& =k_{n}\left[\phi\left(u, J^{-1}\left(\alpha_{0, n} J x_{n}+\sum_{i=1}^{N} \alpha_{i, n} J w_{i, n}\right)\right)\right] \\
& =k_{n}\left[\|u\|^{2}-2\left\langle u, \alpha_{i, 0} J x_{n}+\sum_{i=1}^{N} \alpha_{i, n} J w_{i, n}\right\rangle+\left\|\alpha_{0, n} J x_{n}+\sum_{i=1}^{N} \alpha_{i, n} J w_{i, n}\right\|^{2}\right.
\end{aligned}
$$




$$
\begin{aligned}
\leq & k_{n}\left[\|u\|^{2}-2\left(\left\langle u, \alpha_{0, n} J x_{n}\right\rangle+\left\langle u, \sum_{i=1}^{N} \alpha_{i, n} J w_{i, n}\right\rangle\right)\right. \\
& \left.+\alpha_{0, n}\left\|x_{n}\right\|^{2}+\sum_{i=1}^{N} \alpha_{i, n}\left\|w_{i, n}\right\|^{2}-\alpha_{0, n} \alpha_{i, n} g\left(\left\|J x_{n}-J w_{i, n}\right\|\right)\right] \\
= & k_{n}\left[\alpha_{0, n}\left(\|u\|^{2}-2\left\langle u, J x_{n}\right\rangle+\left\|x_{n}\right\|^{2}\right)+\sum_{i=1}^{N} \alpha_{i, n}\left(\|u\|^{2}-2\left\langle u, J w_{i, n}\right\rangle+\left\|w_{i, n}\right\|^{2}\right)\right. \\
& \left.-\alpha_{0, n} \alpha_{i, n} g\left(\left\|J x_{n}-J w_{i, n}\right\|\right)\right] \\
& =k_{n}\left[\alpha_{0, n} \phi\left(u, x_{n}\right)+\sum_{i=1}^{N} \alpha_{i, n} \phi\left(u, w_{i, n}\right)-\alpha_{0, n} \alpha_{i, n} g\left(\left\|J x_{n}-J w_{i, n}\right\|\right)\right] \\
& \leq k_{n}\left[k_{n} \alpha_{0, n} \phi\left(u, x_{n}\right)+k_{n} \sum_{i=1}^{N} \alpha_{i, n} \phi\left(u, x_{n}\right)-\alpha_{0, n} \alpha_{i, n} g\left(\left\|J x_{n}-J w_{i, n}\right\|\right)\right] \\
= & k_{n}\left[k_{n} \phi\left(u, x_{n}\right)-\alpha_{0, n} \alpha_{i, n} g\left(\left\|J x_{n}-J w_{i, n}\right\|\right)\right] \\
= & k_{n}^{2} \phi\left(u, x_{n}\right)-k_{n} \alpha_{0, n} \alpha_{i, n} g\left(\left\|J x_{n}-J w_{i, n}\right\|\right) .
\end{aligned}
$$

Thus,

$$
\phi\left(u, x_{n+1}\right) \leq k_{n}^{2} \phi\left(u, x_{n}\right)-k_{n} \alpha_{0, n} \alpha_{i, n} g\left(\left\|J x_{n}-J w_{i, n}\right\|\right) .
$$

Hence, we have

$$
k_{n} \alpha_{0, n} \alpha_{i, n} g\left(\left\|J x_{n}-J w_{i, n}\right\|\right) \leq k_{n}^{2} \phi\left(u, x_{n}\right)-\phi\left(u, x_{n+1}\right) .
$$

Since $\left\{\phi\left(u, x_{n}\right)\right\}$ is convergent, we have

$$
\lim _{n \rightarrow \infty}\left(k_{n}^{2} \phi\left(u, x_{n}\right)-\phi\left(u, x_{n+1}\right)\right)=0 .
$$

As, we get $\lim _{n \rightarrow \infty} \alpha_{0, n} \alpha_{i, n}>0$

$$
\lim _{n \rightarrow \infty} g\left(\left\|J x_{n}-J w_{i, n}\right\|\right)=0 .
$$

From the property of $g$, we have

$$
\lim _{n \rightarrow \infty}\left\|J x_{n}-J w_{i, n}\right\|=0 .
$$

Since $J^{-1}$ is uniformly norm-to-norm continuous on bounded sets, we have

$$
\lim _{n \rightarrow \infty}\left\|x_{n}-w_{i, n}\right\|=0 .
$$

Since $\left\{x_{n}\right\}$ is bounded, there exists a subsequence $\left\{x_{n_{k}}\right\}$ of $\left\{x_{n}\right\}$ such that $\left\{x_{n_{k}}\right\}$ converges weakly to $\hat{x} \in C$. From (20) and $F(T)$, we have $\hat{x} \in F(T)$.

Next, we show that $\hat{x} \in \operatorname{GMEP}(f, A, \varphi)$. Let $T=\sup _{n \in \mathbb{N}}\left\{\left\|x_{n}\right\|,\left\|u_{n}\right\|\right\}$ from Lemma 5, and putting $x_{n}=T_{r_{n}} u_{n}$, we have from Lemma $7(d)$ and(19) that for $u \in F(T) \cap$ $\operatorname{GMEP}(f, A, \varphi)$.

$$
\begin{aligned}
g_{1}\left(\left\|x_{n}-u_{n}\right\|\right) & \leq \phi\left(x_{n}, u_{n}\right) \\
& \leq \phi\left(u, u_{n}\right)-\phi\left(u, x_{n}\right) \\
& \leq k_{n} \phi\left(u, u_{n}\right)-\phi\left(u, x_{n}\right) \\
& \leq k_{n}^{2} \phi\left(u, x_{n-1}\right)-\phi\left(u, x_{n}\right) .
\end{aligned}
$$


Since $\left\{\phi\left(u, x_{n}\right)\right\}$ converges, we have

$$
\lim _{n \rightarrow \infty} g_{1}\left(\left\|x_{n}-u_{n}\right\|\right)=0 .
$$

From the property of $g_{1}$, we have

$$
\lim _{n \rightarrow \infty}\left\|x_{n}-u_{n}\right\|=0 .
$$

Since $J$ is uniformly norm-to-norm continuous on bounded sets, we have

$$
\lim _{n \rightarrow \infty}\left\|J x_{n}-J u_{n}\right\|=0 .
$$

From $r_{n} \geq a$, we have

$$
\lim _{n \rightarrow \infty}\left\|\frac{J x_{n}-J u_{n}}{r_{n}}\right\|=0
$$

By $x_{n}=T_{r_{n}} u_{n}$, we have

$$
\tau\left(x_{n}, y\right)+\frac{1}{r_{n}}\left\langle y-x_{n}, J x_{n}-J u_{n}\right\rangle \geq 0, \forall y \in C .
$$

As in the proof of Theorem 1, we have $\hat{x} \in \operatorname{GMEP}(f, A, \varphi)$. Therefore, $\hat{x} \in F(T) \cap$ $\operatorname{GMEP}(f, A, \varphi)$. Let $y_{n}=\Pi_{F(T) \cap G M E P(f, A, \varphi)} x_{n}$. From Lemma 2 and $\hat{x} \in F(T) \cap$ $\operatorname{GMEP}(f, A, \varphi)$, we have

$$
\left\langle y_{n_{k}}-\hat{x}, J x_{n_{k}}-J y_{n_{k}}\right\rangle \geq 0 .
$$

From proposition 17, we also have that $\left\{y_{n}\right\}$ converges strongly to $z \in F(T) \cap$ $\operatorname{GMEP}(f, A, \varphi)$, since $J$ is weakly sequentially continuous, as $k \rightarrow \infty$ we have

$$
\langle z-\hat{x}, J \hat{x}-J z\rangle \geq 0 .
$$

On the other hand, since $J$ is monotone, we have

$$
\langle z-\hat{x}, J \hat{x}-J z\rangle \leq 0 .
$$

Hence by (21) and (22), we have

$$
\langle z-\hat{x}, J \hat{x}-J z\rangle=0 .
$$

From the strict convexity of $E$, we have

$$
z=\hat{x} .
$$

Therefore, $\left\{x_{n}\right\}$ converges weakly to $\hat{x} \in F(T) \cap \operatorname{GMEP}(f, A, \varphi)$, and we have

$$
\hat{x}=\lim _{n \rightarrow \infty} \Pi_{F(T) \cap G M E P(f, A, \varphi)^{x_{n}}} .
$$

\section{Acknowledgements}

Not applicable.

Authors' contributions

All authors contributed equally and significantly in writing this paper. All authors read and approved the final manuscript.

Funding

Not applicable.

Availability of data and materials

Not applicable.

Ethics approval and consent to participate

Not applicable. 


\section{Consent for publication}

Not applicable.

\section{Competing interests}

The authors declare that they have no competing interests.

\section{Author details}

${ }^{1}$ Department of Mathematical Sciences, Bayero University, Kano, Nigeria. ${ }^{2}$ Department of Mathematics, Federal College

of Education, Zaria, Kaduna, Nigeria. ${ }^{3}$ Department of Science and Technology Education, Bayero University, Kano, Nigeria.

${ }^{4}$ Department of Mathematics, Ahmadu Bello University, Zaria, Kaduna, Nigeria.

Received: 2 June 2019 Accepted: 9 October 2019

Published online: 26 October 2019

\section{References}

1. Blum, E., Oettli, W.: From optimization and variational inequalities to equilibrium problems. Math. Students. $\mathbf{6 3}$ $123-145$ (1994)

2. Ali, B., Harbau, M. H.: Convergence theorems for pseudomonotone equilibrium problem, split feasibility problem, and multivalued strictly pseudocontractive mappings. Numer. Funct. Anal. Optim. 40(10), 1194-1214 (2019). https:// doi.org/10.1080/01630563.2019.1599014

3. Combettes, I., Hirstoaga, S. A.: Equilibrium programming in Hilbert spaces. J. Nonlinear. Convex Anal. 6, 117-136 (2005)

4. Moudafi, A.: Second-order differential proximal methods for equilibrium problems. J. Inequal. Pure Appl. Math. 4(1), $1-7$ (2003)

5. Tada, A., Takahashi, W.: Strong convergence theorem for an equilibrium problem and a nonexpansive mapping. In: W. Takahashi, W. Tanaka, T. (Eds), Nonlinear analysis and convex analysis, pp. 609-617. Yokohama Publishers, Yokohama, (2007)

6. Tada, A., Takahashi, W.: Weak and strong convergence theorems for a nonexpansive mapping and equilibium problem. J. Opt. Theory Appl. 133, 359-370 (2007)

7. Takahashi, S., Takahashi, W.: Viscosity approximation methods for equilibrium problem and fixed point problems in Hilbert space. J. Math. Anal. Appl. 331, 506-515 (2007)

8. Takahashi, W., Zembayashi, K.: Strong and weak convergence theorems for equilibrium problems and relatively nonexpansive mappings in Banach spaces. Nonlinear Anal. 70, 45-57 (2009)

9. Chang, S. S., Wang, L., Tang, Y. K., Wang, B., Qin, L. J.: Strong convergence theorems for a countable family of quasi- $\phi$-asymptotically nonexpansive nonself mappings. Appl. Mahts. Comput. 218, 7864-7870 (2012)

10. Deng, C. B., Chen, T., Yin, Y. L.: Strong convergence theorems for mixed equilibrium problem and asymptotically $I-$ nonexpansive mapping in Banach spaces. Abstr. Appl. Anal. 965737, 12 (2014)

11. Ezeora, J. N.: Convergence theorem for generalized mixed equilibrium problem and common fixed point problem for a family of multivalued mappings. Int. J. Anal. Appl. 10(1), 48-57

12. Cioranescu, l.: Geometry of Banach spaces, duality mappings and nonlinear problems. Kluwer, Dordrechi (1990)

13. Alber, Y. I.: Metric and generalised projection operators in Banach spaces, properties and applications. Marcel Dekker, New York (1996)

14. Kamimura, S., Takahashi, W.: Strong convergence of a proximal-type algorithm in a Banach space. SIAM J. Opt. 13, 938-945 (2002)

15. Takahashi, W.: Nonlinear functional analysis. Yokohama Publishers, Yokohama (2000)

16. Matsushita, S., Takahashi, W.: Weak and strong convergence theorems for relatively nonexpansive mappings in Banach space. Fixed Point Theory Appl. 2004, 37-47 (2004)

17. Matsushita, S., Takahashi, W.: A strong convergence theorem for relatively nonexpansive mappings in a Banach space. J. Approx Theory. 134, 257-266 (2005)

18. Reich, S.: A weak convergence theorem for the alternating method with Bregman distance. Marcel Dekker, New York (1996)

19. Chang, S. S., Kim, J. K., Wang, X. R.: Modified block iterative algorithm for solving convex feasibility problem in Banach spaces. J. ineq. Appl. 14 (2010). Article ID 869684

20. Takahashi, W.: Convex analysis and approximation of fixed points. Yokohama Publishers, Yokohama (2000). (in Japanese)

21. Tan, K., Xu, H. K.: Approximation fixed points of nonexpansive mappings by the Ishikawa iteration process. J. Math. Anal. Appl. 178(2), 301-308 (1993)

22. Zhang, S. S.: Generalized mixed equilibrium problem in Banach spaces. Appl. Math. Mech. 30(9), 1105-1112 (2009)

\section{Publisher's Note}

Springer Nature remains neutral with regard to jurisdictional claims in published maps and institutional affiliations. 